\title{
Supplementary material of
}

\section{On the diurnal, weekly, seasonal cycles and annual trends in atmospheric $\mathrm{CO}_{2}$ at Mount Zugspitze, Germany during 1981-2016}

5 Ye Yuan ${ }^{1}$, Ludwig Ries ${ }^{2}$, Hannes Petermeier ${ }^{3}$, Thomas Trickl ${ }^{4}$, Michael Leuchner ${ }^{1,5}$, Cédric Couret ${ }^{2}$, Ralf Sohmer $^{2}$, Frank Meinhardt ${ }^{6}$, Annette Menzel ${ }^{1,7}$

${ }^{1}$ Department of Ecology and Ecosystem Management, Technical University of Munich (TUM), Freising, Germany

${ }^{2}$ German Environment Agency (UBA), Zugspitze, Germany

${ }^{3}$ Department of Mathematics, Technical University of Munich (TUM), Garching, Germany

$10{ }^{4}$ Institute of Meteorology and Climate Research, Atmospheric Environmental Research (IMK-IFU), Karlsruhe Institute of Technology (KIT), Garmisch-Partenkirchen, Germany

${ }^{5}$ Springer Nature B.V., Dordrecht, Netherlands

${ }^{6}$ German Environment Agency (UBA), Schauinsland, Germany

${ }^{7}$ Institute for Advanced Study, Technical University of Munich (TUM), Garching, Germany

15 Correspondence to: Ye Yuan (yuan@wzw.tum.de) 
a)

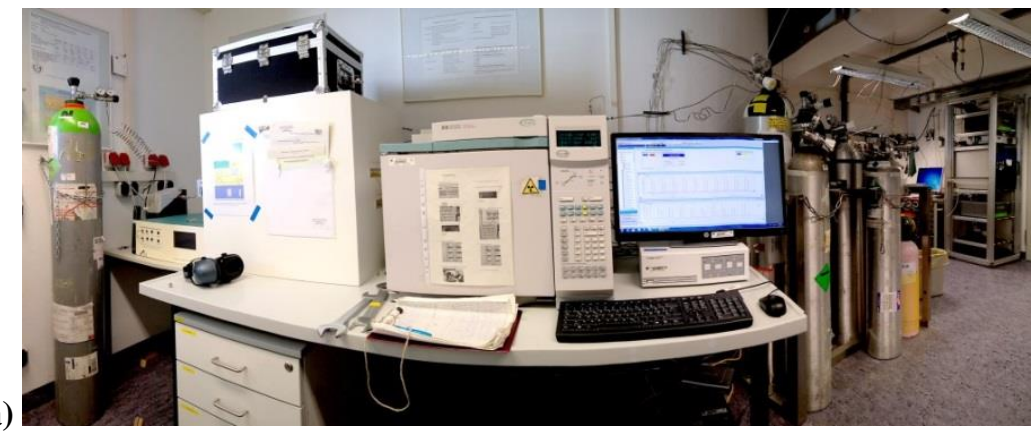

b)

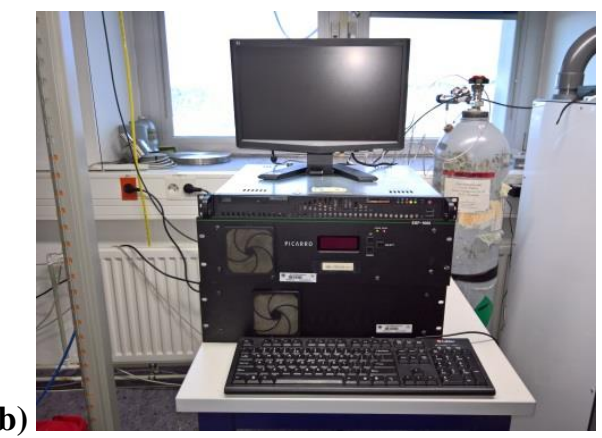

Figure S1: Instrumental setups at ZSF, a) Modified HP 6890 with Gas Chromatography (GC); b) Picarro EnviroSense 3000 i with Cavity Ring-down Spectroscopy (CRDS). 

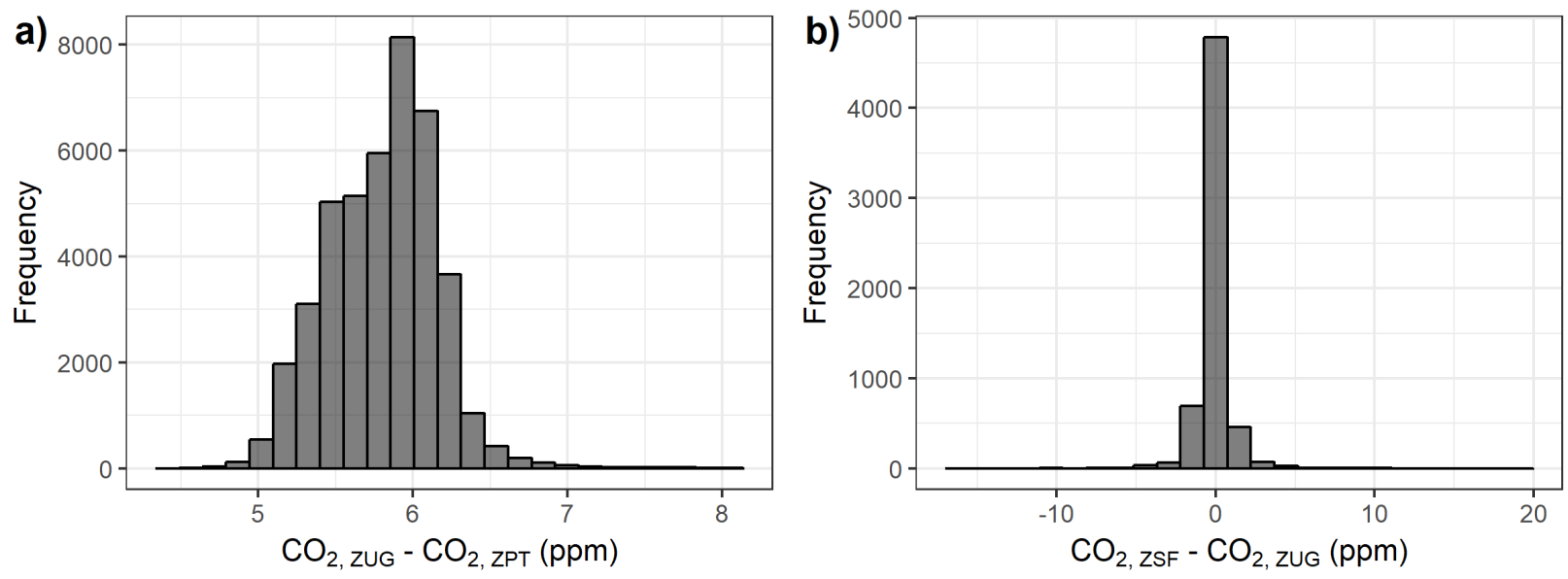

Figure S2: a) Histogram for the offsets between $\mathrm{CO}_{2}$ measurements at ZPT and ZUG for the period of 1995-1997. b) Histogram of the offsets between $\mathrm{CO}_{2}$ measurements at ZUG and ZSF for the period of April to December 2001. 


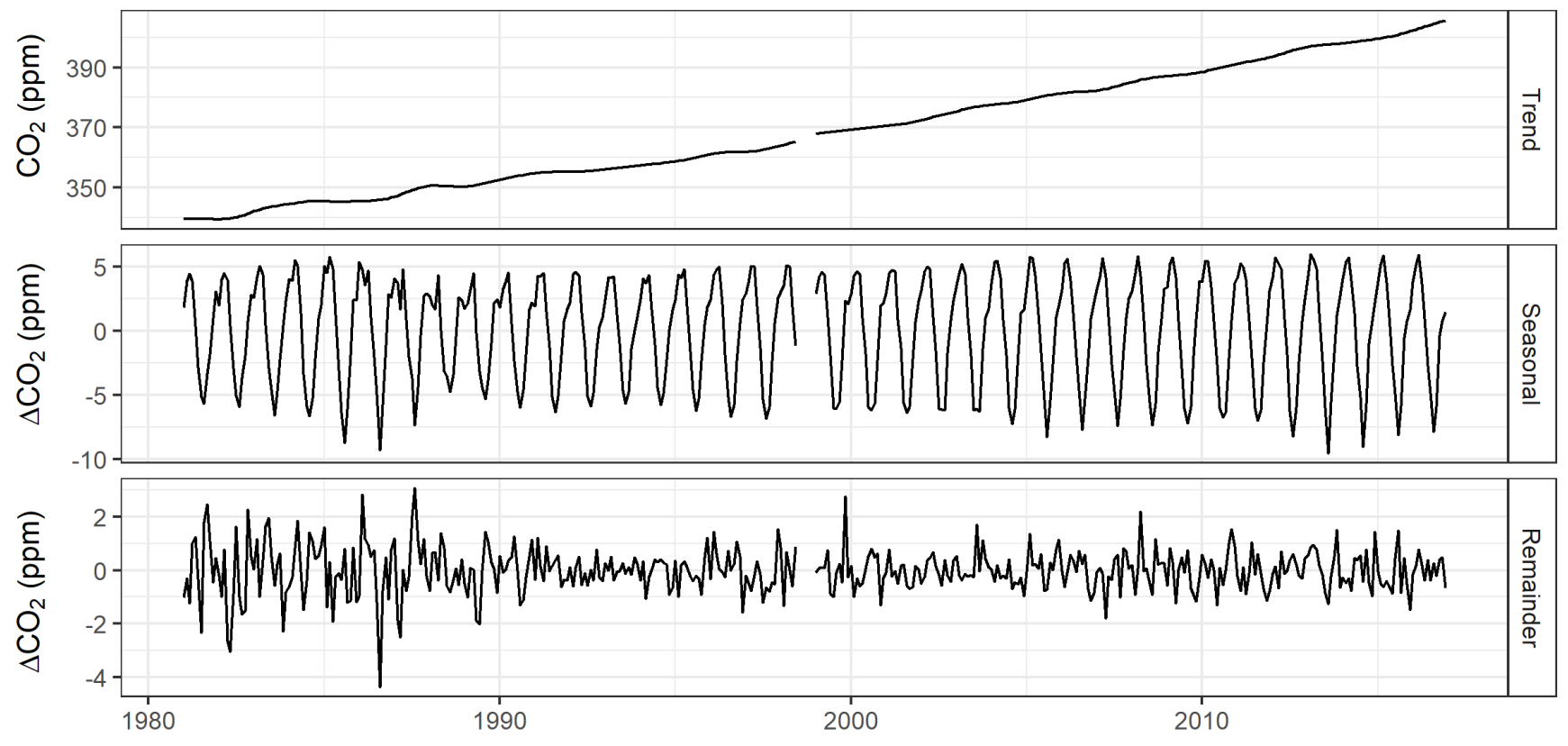

Figure S3: STL-decomposed results of $\mathrm{CO}_{2}$ measurements at Mount Zugspitze sites. 


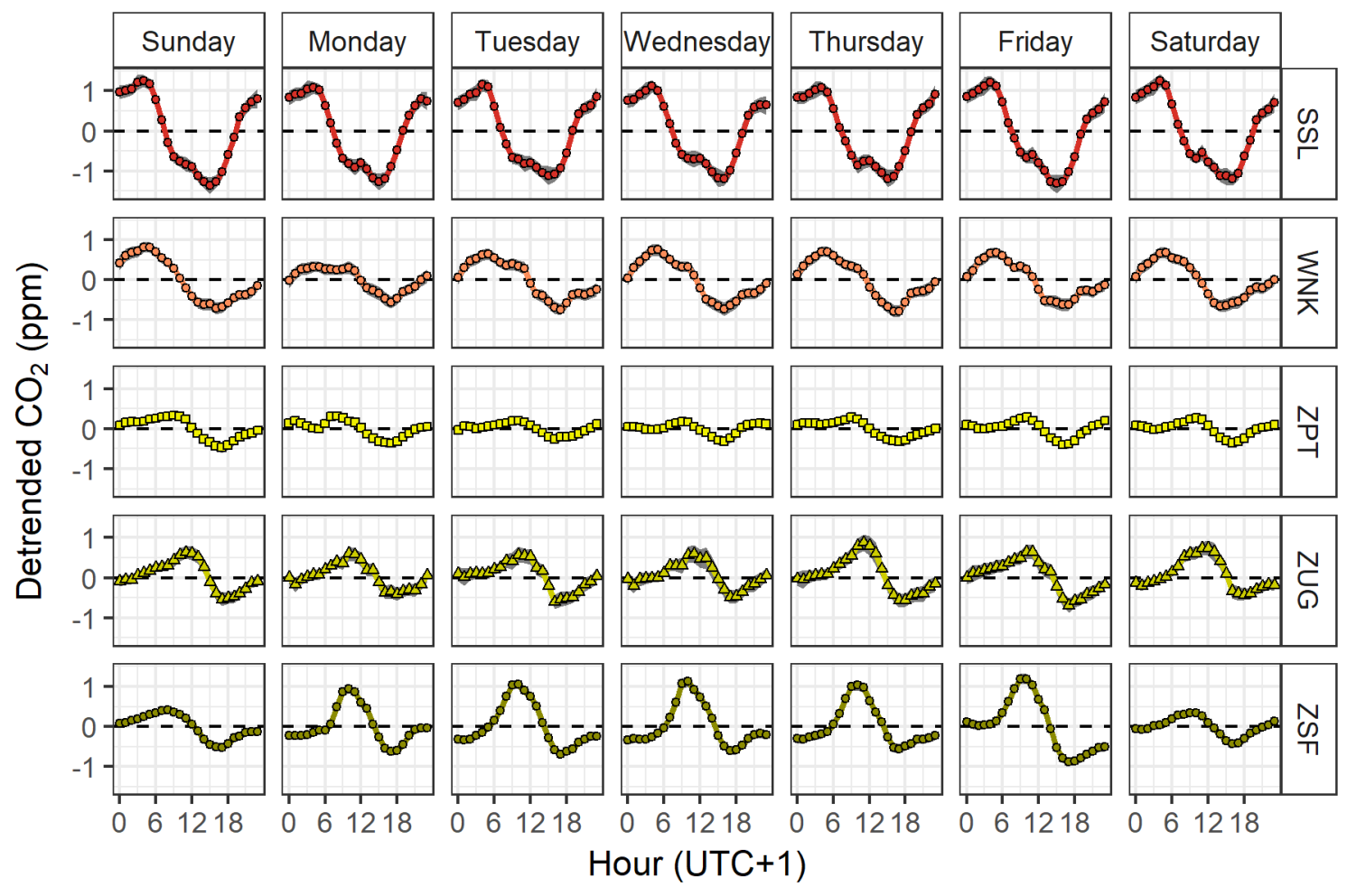

Figure S4: Detrended mean $\mathrm{CO}_{2}$ diurnal cycles by sites and days of the week. Uncertainties of $95 \%$ confidence intervals are shown by the shaded areas. 